\title{
OBSERVATIONS ON RESPIRATION IN ARTICULAR CARTILAGE*
}

BY MORRIS A. BOWIE, OTTO ROSENTHAL AND GEORGE

$$
\text { WAGONER }
$$

(From the Laboratory of Orthopcedic Research, Harrison Department of Surgical Research, Schools of Medicine, University of Pennsylvania, Philadelphia. Aided in part by a grant from the Bryn Mawr Fund for the Investigation and Care of Chronic Arthritis.)

LITTLE was known about the metabolism of articular cartilage till 1936, when Bywaters ${ }^{1}$ published the first quantitative data on the subject. He reported a well-defined glucolysis, equivalent per cell to that of other adult tissues, but was unable to demonstrate an appreciable utilization of oxygen. It seemed improbable that a tissue without any respiratory ability could synthesise the complex constituents of its matrix. Since Bywaters had employed old equine cartilage in his study, reinvestigation of the problem, using cartilage of young as well as of older animals, seeme worth while. Accordingly, bovine articular cartilage securede․ from young, adult, and old animals was employed.

\section{The Respiratory Power of Cartilage Cells in} Different Ages

The comparison of the respiratory power of articular cartilage cells at different ages was based on the manometric determination of the oxygen uptake of surviving cartilage slices in a substratefree phosphate Ringer's solution. Use of the rate of spontaneous oxygen consumption (auto-respiration) as a measure of the respiratory power of cartilage appeared justifiable because of two observations : (1) The constancy of auto-respiration over extended experimental periods indicated that its rate was not limited by a lack of combustible substrates; and (2) the addition of substrates either did not significantly increase or did depress the rate of auto-respiration.

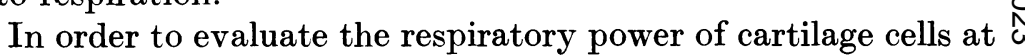
different ages from the measured rate of oxygen uptake per unit

* Received for publication May 17, 1941. 
mass of slices, allowance had to be made for the decrease in the cell content of the slices with ageing. It was shown by means of cell counts ${ }^{2,6}$ that the rate of anaerobic glucolysis, which is readily demonstrable manometrically, was proportional to the cell content of the slices in all the ages studied. Hence, the ratio

(rate of auto-respiration)

(rate of anaerobic glucolysis) $\times 100$

could be used as an estimate of the respiratory power of the cartilage cells.

The ratio of auto-respiration to glucolysis amounted to $8: 1$ in calves under half a year of age to $5: 7$ in adult animals one to seven years old and to $3: 1$ in animals eight years and older. The differences between the mean ratios obtained in the three age groups were statistically significant.

The ratio of auto-respiration to glucolysis in old bovine articular cartilage was in close agreement with Bywaters' data for equine material. The ratio in calf cartilage, however, was two and a half times greater than Bywaters' figure. Thus, our experimental data indicated that young cartilage cells were endowed with a small, but well-defined respiratory power in addition to their glycolytic capability. In contrast to the glycolytic power, however, the respiratory power of the cartilage cell decreased by 60 per cent. during the process of maturation and ageing.

\section{The Properties of Respiration in Calves' Cartilage}

The data of Table I indicate that the respiratory quotient of calves' cartilage in a substrate-free medium was compatible with the combustion of either protein and fat or of carbohydrate and fat. Bywaters obtained an R.Q. of 0.71 for synovial membrane, suggesting oxidation of fat alone.

A peculiarity of cartilage respiration consisted in the marked depressing action which glucose exerted upon the respiratory rate. We suggested ${ }^{3}$ as one possible explanation that the utilization of the added glucose by cartilage cells prevented the combustion of certain cellular substrates. Particularly, cellular protein might be conserved since the protein-sparing action of glucose has long been recognised.

Because of the high aerobic glucolysis in articular cartilage it seemed evident that a glycolytic utilization of glucose might be 
responsible for the depressing action of this hexose on respiration. However, the significant increment of the R.Q. by the addition of glucose to cartilage ( $c f$. Table I) demonstrated that an oxidative utilization of the hexose took place simultaneously with its glycolytic breakdown.

The results of experiments summarised in Table II showed that the addition of iodoacetate in a concentration that inhibited glucolysis by about 95 per cent. virtually abolished the depressing action of glucose on the respiratory rate. This effect of iodoacetate strongly suggested that the depressing action of glucose on cartilage respiration was linked with the glycolytic splitting of the hexose.

In order to secure more information on the type of combustions which were started after the blocking of glycosis, we determined the R.Q. of cartilage slices in the presence of iodoacetate. Table II shows that under these conditions the R.Q. still remained close to one. This order of magnitude of the R.Q. values demonstrated that fat combustion did not play an essential rôle in articular cartilage even if carbohydrate utilisation had been greatly limited. The values were compatible with the oxidation of protein to water, carbon dioxide, and ammonia, a process which could produce R.Q. values of 0.95 . This interpretation is to be regarded as tentative, since the ammonia metabolism of the cartilage cell was not studied.

At present it cannot be excluded that respiration in the presence of iodoacetate was based upon the oxidation of small amounts of lactic acid which were either preformed or produced by the cells in spite of the presence of the drug. The persistence of only 3 per cent. of the glycolysis would be sufficient to yield the amount of lactic acid required for the respiration observed.

An oxidation of lactic acid by articular cartilage was indicated by the fact that the addition of lactate increased the rate of respiration if the new formation of lactic acid from cellular carbohydrate was restricted by the presence of iodoacetate. Indications for the oxidations of pyruvic and succinic acids have been previously reported. ${ }^{3}$

The experimental results discussed above justify the conclusion that the young cartilage cell is capable of completely oxidising carbohydrate and, possibly, protein. 


\section{Dehydrogenase Systems in Articular Cartilage}

Bywaters in $1936^{1}$ noted that the addition of methylene blue to equine articular cartilage markedly increased the rate of oxygen consumption. He pointed out that articular cartilage was apparently endowed with dehydrogenatic enzymes, but that the function of this substrate-activating group of the respiratory enzymes in a tissue devoid of respiratory power remained a mystery.

The intensity of the dye-stimulated respiration in bovine articular cartilage facilitated a more detailed study on the nature of the dehydrogenatic processes. The R.Q. of the dyestimulated respiration $(0.865 \pm 0.027)$ had an order of magnitude similar to that of the auto-respiration of calves' cartilage. ${ }^{4}$ The rate of the dye-stimulated respiration was slightly but definitely increased by the addition of lactate or pyruvate and sustained by the addition of succinate. Thus, the reactions which were accomplished by the joint action of the cellular dehydrogenases and the artificial oxidation-reduction dye had the same character as the natural cartilage respiration, where the oxygen-activating group of the respiratory enzymes took the place of the experimental redox dye. This qualitative similarity between the dye-stimulated and the spontaneous respirations demonstrated directly the function of the dehydrogenases in cartilage metabolism.

In contrast to the spontaneous respiration of articular cartilage the dye-stimulated respiration was markedly increased by the addition of glucose. Like the depressing effect of glucose on the spontaneous respiration, the accelerating action of the hexose on the dye-stimulated respiration was completely abolished when glucolysis was inhibited by iodoacetate.

It is known that the transformation of triose-phosphate to phosphoglycerate-the oxidative step of the glycolytic oxidationreduction of glucose to lactic acid-is an enzymatic reaction which is especially sensitive to iodoacetate poisoning. In view of the equally great sensitivity to iodoacetate of both the anaerobic and the aerobic glucose utilization in articular cartilage it seemed highly probable that the system triose-phosphate dehydrogenase+co-enzyme 1 participated in the normal glucose respiration as well as in the oxidation of glucose by methylene blue. It is possible that at this point of the intermediary glucose 
metabolism the glucolytic and oxidative pathways diverged. The subsequent stages of the glucose oxidation by methylene blue, a combustion which was apparently not complete, ${ }^{4,5}$ remain to be studied.

Thus far analysis of the nature of the dye-stimulated respiration in articular cartilage has provided indications that dehydrogenase systems concerned with the oxidation of glucose, lactate, pyruvate, and succinate are present. Since all of these substances were metabolised by calves' cartilage in the absence of dye, the rôle of dehydrogenatic enzymes in the metabolism of articular cartilage appears understandable.

\section{Dehydrogenatic Power of Cartilage Cells in Different Ages}

The decline of the respiratory power of ageing cartilage cells raised the question as to whether the whole or only one group of the respiratory enzymes diminished in amount with the progress of age. Changes in the amount of dehydrogenases-i.e., in the substrate-activating group of respiratory enzymes-were most suitable for a quantitative estimate, since the rate of the dyestimulated respiration provided a measure of the dehydrogenatic capacity of cartilage in the different ages.

The addition of methylene blue to surviving slices of cartilage increased the initial rate of oxygen consumption three-fold in calves, ten-fold in adult animals, and twenty-fold in old animals. The extent of the stimulative effect of methylene blue in the age groups showed that only a fraction of the total dehydrogenatic capacity of the cells was utilized in normal respiration and that this utilized fraction diminished in ageing cells. Because of this excess of dehydrogenatic capacity over total respiratory capacity in all the ages studied, it seemed likely that the declining respiratory power of the ageing cells was due to a deterioration of their oxygen-activating power.

For a quantitative comparison of the dehydrogenatic power in the age groups the rate of the dye-stimulated respiration in a substrate-free medium was of limited value, because changes of the rate incident to ageing might depend not only upon differences in the enzyme content of the tissue, but also upon variations in the amount of cellular substrates. It seemed more accurate, therefore, to restrict the comparison to one single dehydrogenatic process, such as glucose dehydrogenation, where the substrate 
concentration could be kept under experimental control. The increment of the rate of the dye-stimulated respiration due to the addition of glucose was employed as a measure of the rate of glucose dehydrogenation. The ratio of this increment to the rate of anaerobic glycolysis served as an estimate of the glucosedehydrogenatic power per cell. ${ }^{6}$

The ratio of glucose dehydrogenation to glucolysis increased by 11 per cent. from infancy to adolescence and decreased by 23 per cent. from infancy to old age. The changes of the ratio with age were, however, not statistically significant.

This result lent support to the view that the dehydrogenatic power of the cartilage cell changed very little, if at all, during the process of ageing. Thus, it seemed highly probable that the deterioration of the respiratory power in ageing articular cartilage could be ascribed to a gradual loss of components of the oxygenactivating group of the respiratory enzymes.

\section{The Inter-relationship of Degeneration, Ageing, and}

\section{Metabolic Activity}

Bauer and Bennett have shown that alterations in articular cartilage, characteristic of degenerative or hypertrophic arthritis, occur in all cattle over two years of age.? In young adult cattle the degenerative changes consist in circumscribed lesions on the proximal surfaces of the metacarpals. With advancing age, additional joints become affected and general degenerative alterations in cells and matrix accompany the localised defects The decline in the respiratory power of articular cartilage was recognizable at the same age period when the first arthritic phenomena became apparent and the decline progressed with the extension of the degenerative alterations.

The diminution in the respiratory power of articular cartilage should not be considered a consequence of the degenerative alterations, because it is found in the cartilage from joints where both macroscopic and microscopic alterations are still absent. Obviously, the loss in metabolic activity is correlated with the chronological and structural age of the cartilage, and precedes the visible manifestations of the process of ageing. The gradual decline of the respiratory power might be denoted as the metabolic equivalent of the process of ageing in articular cartilage. In view of the fact that degenerative arthritis (hypertrophic or osteo-arthritis) is a disease of the more advanced age groups in 
man, the gradual loss in the respiratory power of articular cartilage is possibly an intrinsic factor in the development of degenerative arthritis.

\section{Conclusions}

Articular cartilage of calves exhibits a small but well-defined respiratory activity. The young cartilage cell is capable of oxidising carbohydrate and, perhaps, protein.

The respiratory power of bovine articular cartilage cells decreases by 30 per cent. from infancy to adolescence and by 60 per cent. from infancy to old age. This decrease is probably due to a gradual loss of components of the oxygen-activating group of the respiratory enzymes, because the activity of the dehydrogenatic group of these enzymes remained virtually constant throughout the age groups tested.

In view of the parallelism between the decrease of respiratory power and the appearance of degenerative alterations in ageing bovine cartilage it is suggested that a gradual loss of the respiratory power of the cartilage contributes to the higher frequency of degenerative joint diseases in the more advanced age groups of man.

It remains for further study to find out if and how the cartilage cell utilizes its respiratory capability for the synthesis of specific constituents of cartilage tissue, and to elucidate the nature of the enzymatic components which diminish in amount with the progress of age.

Table I.-The Influence of Glucose upon the Respiratory Rate and Upon the Respiratory Quotient of Calves' Cartilage.

Gas: Air. Medium: Phosphate Ringer's. $\mathrm{pH}: \mathbf{7 \cdot 4}$. Respiratory rate: $1 \mu \mathrm{O}_{2}$ consumed per mg. dry weight of cartilage per hour. Experimental period: Three hours for the determination of rates, four hours for R.Q. measurements.

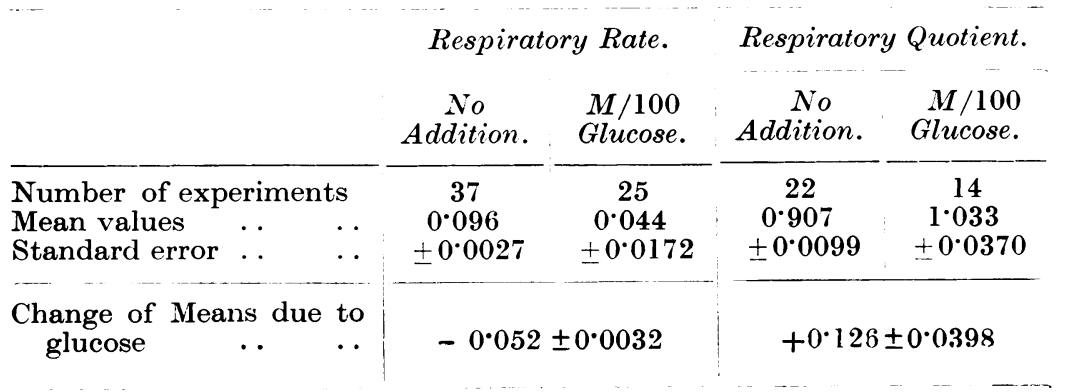


Table II.-Action of Iodoacetate on Cartilage Respiration. Experimental period: Four hours.

\begin{tabular}{|c|c|c|c|c|c|c|}
\hline \multirow{3}{*}{ Additions. } & \multirow[b]{3}{*}{ Rate. } & \multirow[b]{3}{*}{$R . Q$. } & \multicolumn{4}{|c|}{$+M / 10,000$ Iodoacetate. } \\
\hline & & & & & $M / 100$ & Glucose. \\
\hline & & & Rate. & $R . Q$. & Rate. & $R . Q$. \\
\hline $\begin{array}{l}\text { Number of ex- } \\
\text { periments } \\
\text { Mean values } \\
\text { Standard error }\end{array}$ & $\begin{array}{c}10 \\
0.095 \\
+0.0038\end{array}$ & $\begin{array}{c}8 \\
0.886 \\
\pm 0.0217\end{array}$ & $\begin{array}{c}10 \\
0.094 \\
\pm 0.0045\end{array}$ & $\begin{array}{c}8 \\
0.959 \\
\pm 0.0213\end{array}$ & $\begin{array}{c}10 \\
0.090 \\
+0.0036\end{array}$ & $\begin{array}{c}10 \\
0.991 \\
\pm 0.0149\end{array}$ \\
\hline
\end{tabular}

\section{REFERENCES}

1. Bywaters, E. G. L.: "Reports on Chronic Rheumatic Diseases," 1936, No. $2,104$.

2. Rosenthal, O., Bowie, M. A., and Wagoner, G.: Journ. Cell. and Comp. Physiol., 1941, xvii. 221.

3. Rosenthal, O., Bowie, M. A., and Wagoner, G.: Science, 1940, xcii. 382.

4. Rosenthal, O., Bowie, M. A., and Wagoner, G.: Journ. Cell. and Comp. Physiol., in preparation, 1940.

5. Hills, G. M.: Biochem. Journ., 1940, xxxiv. 1070.

6. Wagoner, G., Rosenthal, O., and Bowie, M. A.: Amer. Journ. Med. Sci., 1941, cci. 489.

7. Bauer, W., ANd Bennett, G. A.: Journ. Bone and Joint Surg., 1936, xviii. 1. 